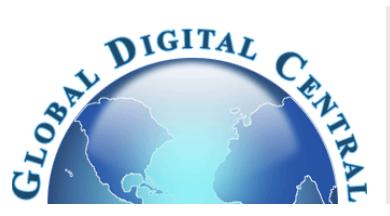

\title{
THERMOPHORESIS AND BUOYANCY EFFECTS ON CHEMICALLY REACTIVE UPPER CONVECTED MAXWELL FLUID FLOW INDUCED BY AN EXPONENTIALLY STRETCHING SHEET: APPLICATION OF CATTANEO-CHRISTOV HEAT FLUX
}

\author{
N.Vijaya ${ }^{\mathrm{a},{ }^{*}}$, P. Krishna Jyothi ${ }^{\text {b }}$, A. Anupama ${ }^{\mathrm{c}}$, R. Leelavathi ${ }^{\mathrm{d}}$, K. Ambica ${ }^{\mathrm{e}}$, \\ ${ }^{a}$ Department of Mathematics, Koneru Lakshmaiah Education Foundation, Vaddeswaram, Guntur, AP, India-522502 \\ ${ }^{b}$ Department Mathematics, Annamacharya Institute of Technology and Sciences, Tirupati, AP, India -517520 \\ ${ }^{c}$ Department of Mathematics, DVR \&Dr. HS MIC College of Technology, Kanchikacherla, Krishna, AP, India-521180 \\ ${ }^{d}$ Department of Mathematics, Andhra Loyola Institute of Engineering and Technology, Vijayawada, AP, India-520008 \\ ${ }^{e}$ Department of Mathematics, CMR Engineering College, Kandlakoya(v), Hyderabad, Telangana, India-501401
}

\begin{abstract}
The main intention of this study is to explore Maxwell fluid under the influence of thermophoresis and buoyancy forces induced by exponentially stretching sheet under chemical reaction. Cattaneo-Christov heat flux model is used to explore heat and mass characteristics with variable magnetic field, and chemical reaction. Variables of similarity were induced to transmute partial differential equations into dimensionless equations and are resolved numerically by elegant method bvp 4c. Behavior of various critical parameters on velocity, temperature and concentrations is graphically presented and discussed. Non Newtonian nature of the Maxwell fluid is clearly explored by the Maxwell parameter, it was found that for higher values of Maxwell parameter velocity profiles decreases. Thermal and solutal buoyancy forces acts in favor to velocity and thermophorotic parameter acts against concentration. Impact of Skin friction, Sherwood and Nusselt numbers on the flow configurations for diverse critical parameters are exposed realistically via graphs. Arithmetical results that obtained in the current exploration are confirmed with previously explored values in very marginal way.
\end{abstract}

Key words: Maxwell fluid, Cattaneo Christov heat flux model, Buoyancy forces, Upper Convection

\section{INTRODUCTION}

MHD flow intensifies the curiosity of many researchers in modern era for the reason that it plays immense role in numerous applications in all fields of science and technology like plasma physics, aerodynamics, astrophysics and super conduction coils, polyethylene industries, coatings, biomedical applications, drug transportations and so forth. Numerous classical theories and transport models of heat and mass transfer are available in the literature. In these models both thermal and concentration relaxation features were involved. Fourier (1822) and Fick (1855) described the phenomenon of heat and mass transfer. The main inadequacy of the Fourier's law known as "Paradox of heat conduction" is that it leads the energy equation to a parabolic equation. To overcome this paradox, several modified versions of the Fourier's law have been introduced. Cattaneo (1948) in his experiment included heat flux relaxation time required to establish steady conduction once a temperature gradient is imposed. Later Christov (2009) proposed a modification to the time derivative in the Maxwell-Cattaneo model with the Oldroyd upper-convected derivative preserving material invariant formulation. Hayat et al. (2016) studied three -dimensional flow of nanofluid with Cattaneo-Christov double diffusion under Brownian motion and thermophoresis effects. Sui et al.

(2016) in their paper reported that higher values of slip parameter decelerates velocity and skin friction. Awais et al. (2018) analyzed that heat transfer rate decays for advanced relaxation time whereas it increases for higher Prandtl number.

The viscosity of the fluids like paints, greases, lubricant oils coal tar, jellies, and paste varies depending upon the influences like shear in fluid, pressure and temperature. These fluids are non-Newtonian in nature. In view of various rheological properties of non-Newtonian fluids several constitutive relationships between stress and rate of shear are examined. Fluids of non-Newtonian types are mainly distributed among integral, rate and differential types. Maxwell fluid falls under rate type non-viscous fluids category. This class illustrates the relaxation time effects. Fetecau (2003) obtained exact solution for Maxwell fluid flow. Umer Farooq et al .(2019) considered Buongiorno model to explore properties of Maxwell fluid with nanomaterial over stretching surface. Wubshet Ibrahim et al. (2020) scrutinized upper convected slip effects of stagnation point flow of 
Maxwell fluid. Vajravelu et al. (2017), Rahbari et al. (2018), Gireesha et al. (2018), scrutinized non-Newtonian Maxwell fluid with different physical conditions such as viscous dissipation, Newtonian heating, homogeneous-heterogeneous chemical reactions, and thermal stratification past different stretching surfaces. Their result shows that as Prandtl number increases, temperature, as well as rate of heat transfer, dwindled.

Chemical reaction with mixed convection plays dynamic role in many industrial and technological applications, so there is great demand to minimize number of reagents and to maximize desired output. Concentration and temperature differences or both effects influence rate of heat and mass transfer. Exothermic and endothermic chemical reactions added to combustion is to be studied thoroughly. Many researchers anticipated to study these effects by considering buoyancy forces through thermal Grashof number, and solutal Grashof number. Transport phenomena due to stretching sheets have superficial role in various engineering applications like paper production, aerodynamic extrusion of sheets, drawing of plastics, cooling of metallic sheets in baths and many others. At present, researchers have anticipated through several experimental studies that desired output from industrial objects can be achieved by varying velocity in different ways. After a thorough survey, they reported that the velocity may be sinusoidal, exponential or nonlinear. Vedavati et al. (2017) showed their interest to study non Newtonian fluids and explored non-Newtonian nature by considering chemical reaction mixed convective conditions and different physical parameters. Rout et al (2013) studied convective surface boundary conditions of moving vertical flat plate. Vijaya et al. (2019, 2020a, 2020b) comprehensively studied Casson fluid flows on stretching surface by considering the impact of mixed convection, radiation, thermophoresis and chemical reactions. Yahaya Shgiya Daniel et al. (2015) investigated the theoretical influence of buoyancy and thermal radiation on MHD flow over stretching sheet. Aswin kumar et al. (2018) reported buoyancy forces on the boundary layer along with radiation and absorption effects. Shateyi (2008) analyzed buoyancy effect on MHD flows by considering suction and blowing. Unsteady magnetohydrodynamic (MHD) flow of pseudoplastic nanofluid in a finite thin film towards a stretching surface with internal heat generation is explored analytically by Lin et al. (2015), Ramesh et al. (2012) scrutinized the magnetohydrodynamic (MHD) stagnation point flow of dusty liquid towards a porous stretched surface with non-uniform heat source/sink.

Thermophoresis is an important physical formation of colloidal particles in a solution which responds to temperature gradient. This can be observed in vaporizer mixtures (fog, dust, geyser steam, and smoke) and in many industrial applications like optical fiber, electronic chip industries, nuclear power projects and so forth. Loganathan et al. (2010) explored the consequences of thermophoresis and injection/suction on the non-Darcy flow of fluid past a wedge in the presence of the magnetic field. Hady et al. (2011) considered the effect of heat sink/source to inspect the behavior of the natural convective flow of nanofluid through a cone. Rawat et al. (2018) accounts for the consequence of slip, Brownian motion, and thermophoresis to investigate the boundary layer flow of $\mathrm{Cu}-\mathrm{H} 2 \mathrm{O}$ nanofluid. Nagalakshmi et al. (2020) elaborately studied Carreau Nanofluid explored using CNT over a nonlinear stretched sheet. To the best of the author knowledge influence of thermophoresis on chemically reactive Maxwell fluid flow with Cattaneo-Christov heat flux model subject to a magnetic field is yet to be examined. The major objective of this study is to explore the effect of thermophoresis, buoyancy effects combined with chemical reaction and magnetic effects on Maxwell fluid over a surface with exponentially stretching sheet. Graphical portrayal is depicted using the powerful tactic bvp4c via MATLAB software.

\section{FLOW ANALYSIS AND MATHEMATICAL MODELLING}

A two dimensional mixed convective flow of an upper convected Maxwell fluid over an elastic sheet is located at $\mathrm{y}=0$, as shown in Fig. 1. In this flow geometry, origin is taken as the fixed point and the pane is elongated along $\mathrm{x}$-axis in its own plane with the velocity as well as $\mathrm{y}$-axis with the velocity $U_{w}(x)=U_{0} e^{\frac{x}{L}}$ in which $U_{0}$ is considered as reference temperature and $\mathrm{L}$ is reference length. A variable magnetic field $B(x)=B_{0} e^{\frac{x}{2 L}}$ is applied normal to the sheet, $B_{0}$ being a constant. The very slight induced magnetic field generated due to electrical fluid is ignored in this study. In this flow pattern $T_{w}=T_{\infty}+T_{0} e^{\frac{A x}{2 L}}$ is variable surface temperature distribution and $C_{w}=C_{\infty}+C_{0} e^{\frac{A x}{2 L}}$ is solute concentration distribution, where $\mathrm{A}$ is exponent constant.

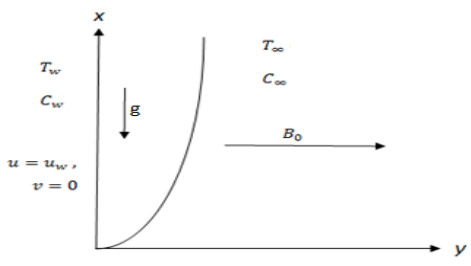

Fig.1. Geometrical outline of the flow

The governing equations of the flow are prearranged here under with terms mentioned in nomenclature considering Boussinesq's approximations with thermal diffusivity $\alpha=\frac{k}{\rho c_{p}}$, and with the thermophoretic velocity

$$
\begin{gathered}
V_{T}=-k_{r} \frac{v}{T_{r}} \frac{\partial T}{\partial y} . \\
\frac{\partial \bar{u}}{\partial x}+\frac{\partial \bar{v}}{\partial y}=0
\end{gathered}
$$

$\bar{u} \frac{\partial \bar{u}}{\partial x}+\bar{v} \frac{\partial \bar{u}}{\partial y}+\lambda\left(\bar{u}^{2} \frac{\partial^{2} \bar{u}}{\partial x^{2}}+\bar{v}^{2} \frac{\partial^{2} \bar{u}}{\partial y^{2}}+2 \bar{u} \bar{v} \frac{\partial^{2} \bar{u}}{\partial x \partial y}\right)=\bar{v} \frac{\partial^{2} \bar{u}}{\partial y^{2}}-\frac{\sigma B^{2}}{\rho}(\bar{u}+$ $\left.\lambda \bar{v} \frac{\partial \bar{u}}{\partial y}\right)+g \beta_{T}\left(T^{*}-T_{\infty}\right)+g \beta_{C}\left(C^{*}-C_{\infty}\right)$

$\alpha \frac{\partial^{2} T^{*}}{\partial y^{2}}=\bar{u} \frac{\partial T^{*}}{\partial x}+\bar{v} \frac{\partial T^{*}}{\partial y}+\lambda_{1}\left[\left(\bar{u} \frac{\partial \bar{u}}{\partial x}+\bar{v} \frac{\partial \bar{u}}{\partial y}\right) \frac{\partial T^{*}}{\partial x}+\left(\bar{u} \frac{\partial v}{\partial x}+\bar{v} \frac{\partial v}{\partial y}\right) \frac{\partial T^{*}}{\partial y}+\right.$ $\left.u^{2} \frac{\partial^{2} T^{*}}{\partial x^{2}}+\bar{v}^{2} \frac{\partial^{2} T^{*}}{\partial y^{2}}+2 \bar{u} \bar{v} \frac{\partial^{2} T^{*}}{\partial x \partial y}\right]$

$\bar{u} \frac{\partial C^{*}}{\partial x}+\bar{v} \frac{\partial C^{*}}{\partial y}=D \frac{\partial^{2} C^{*}}{\partial y^{2}}-\frac{\partial}{\partial y}\left[V_{T}\left(C^{*}-C_{\infty}\right)\right]-k_{0}\left(C^{*}-C_{\infty}\right)$

Under the following boundary conditions

$$
\begin{gathered}
\left\{\begin{array}{c}
\bar{u}=U_{w}(x)=U_{0} e^{\frac{x}{L}} \\
\bar{v}=0 \\
T^{*}=T_{w}(x)=T_{\infty}+T_{0} e^{\frac{A x}{2 L}} \text { at } \mathrm{y}=0 \\
C^{*}=C_{w}(x)=C_{\infty}+C_{0} e^{\frac{A x}{2 L}}
\end{array}\right. \\
\left\{\begin{array}{l}
\bar{u} \rightarrow 0, \\
T^{*} \rightarrow T_{\infty} \\
C^{*} \rightarrow C_{\infty}
\end{array} \text { as } y \rightarrow \infty\right.
\end{gathered}
$$

\section{METHOD OF SOLUTION}

Following likeness variables are introduced to convert partial differential equations (1-4) into ordinary differential equations

$$
\begin{aligned}
& \eta=\left(\frac{U_{0}}{2 v L}\right)^{\frac{1}{2}} \exp \left(\frac{x}{2 L}\right) \\
& \bar{u}=U_{0} \exp \left(\frac{x}{L}\right) f^{\prime}
\end{aligned}
$$




$$
\begin{aligned}
\bar{v} & =-\left(\frac{v U_{0}}{2 L}\right)^{\frac{1}{2}} \exp \left(\frac{x}{2 L}\right)\left(f+\eta f^{\prime}\right) \\
T^{*} & =T_{\infty}+\left(T_{w}-T_{\infty}\right) \theta \\
C^{*} & =C_{\infty}+\left(C_{w}-C_{\infty}\right) \phi
\end{aligned}
$$

Using equations (7 - 9) into equations $(2-4)$, we obtain

$f^{\prime \prime \prime}-2 f^{\prime 2}+f f^{\prime \prime}+\beta\left(3 f f^{\prime} f^{\prime \prime}+\frac{\eta}{2} f^{\prime 2} f^{\prime \prime}-\frac{1}{2} f^{2} f^{\prime \prime \prime}-2 f^{3}\right)+$

$R(\theta+N \phi)-M\left(f^{\prime}-\beta f f^{\prime \prime}\right)=0$,

$\frac{1}{P r} \theta^{\prime \prime}+f \theta^{\prime}+\frac{\beta_{1}}{2}\left(A f f^{\prime \prime} \theta-A(2+A) f^{\prime 2} \theta+(1+2 A) f f^{\prime} \theta^{\prime}-\right.$

$\left.f^{2} \theta^{\prime \prime}\right)-A f^{\prime} \theta=0$,

$\phi^{\prime \prime}+S c\left(f \phi^{\prime}-\tau\left(\theta^{\prime} \phi^{\prime}+\phi \theta^{\prime \prime}\right)-A f^{\prime} \phi-\gamma \phi\right)=0$.

Under the boundary conditions

$$
\begin{gathered}
f(0)=0 \\
f^{\prime}(0)=1 \\
\theta(0)=1 \\
\phi(0)=1 \\
\left\{\begin{array}{l}
f^{\prime}(\infty) \rightarrow 0 \\
\theta(\infty) \rightarrow 0 \\
\phi(\infty) \rightarrow 0
\end{array}\right.
\end{gathered}
$$

Where the dimensionless critical parameter are $\beta=\frac{\lambda U_{0} e^{\frac{x}{L}}}{L}$ (Maxwell parameter), $M=\frac{2 L \sigma B_{0}^{2}}{\rho u_{0}}$ (Magnetic field parameter), $R=\frac{G r_{x}}{R e_{x}^{2}} \quad$ (Thermal buoyancy parameter), $N=\frac{G c}{G r_{x}}$ (Solutal buoyancy parameter), $G r_{x}=$ $\frac{2 g \beta_{T}\left(T_{w}-T_{\infty}\right) L x^{2}}{v^{2}}$ (Grashof number due to temperature), $G c=$ $\frac{2 g \beta_{C}\left(C_{w}-C_{\infty}\right) L x^{2}}{v^{2}}$ (Grashof number due to concentration), $\beta_{1}=\frac{\lambda_{1} U_{0} e^{\frac{x}{L}}}{L}$ (Nondimensional thermal relaxation time), $\operatorname{Pr}=\frac{v}{\alpha} \quad$ (Prandtl number),$S c=\frac{v}{D}$ (Schmidt's number), $\gamma=\frac{2 L k_{0}}{e^{\frac{x}{L} u_{0}}} \quad$ (Chemical reaction parameter), $\tau=$ $-\frac{k_{r}\left(\mathrm{~T}_{\mathrm{w}}-\mathrm{T}_{\infty}\right)}{\mathrm{T}_{\mathrm{r}}}$ (Thermophoretic parameter) .

\section{PHYSICAL QUANTITIES}

The significant engineering physical quantities in this problem are $\mathrm{C}_{\mathrm{f}}$ (=skin friction coefficient), $\mathrm{Nu}_{\mathrm{x}}$ (=local Nusselt number), $\mathrm{Sh}_{\mathrm{x}}$ (=local Sherwood number) respectively are defined below with $R e_{x}=\frac{u_{w} x}{v}$ as local Reynolds number

$C_{f}=\frac{\tau_{w}}{\rho u_{w}^{2}}, \quad N u_{x}=\frac{x q_{w}}{k\left(T_{w}-T_{\infty}\right)}, S h_{x}=\frac{x m_{w}}{D\left(C_{w}-C_{\infty}\right)}$,

where the wall shear stress $\tau_{w}$, the surface heat flux $q_{w}$ and mass flux $m_{w}$ are given by

$\tau_{w}=\mu(1+\beta)\left(\frac{\partial \bar{u}}{\partial y}\right)_{y=0}, q_{w}=-k\left(\frac{\partial T^{*}}{\partial y}\right)_{y=0}, m_{w}=-D\left(\frac{\partial C^{*}}{\partial y}\right)_{y=0}$

Making use of equation (18) in equation (17), we obtain

$C_{f} \sqrt{R e_{x}}=(1+\beta) f^{\prime \prime}(0), \frac{N u_{x}}{\sqrt{R e_{x}}}=-\theta^{\prime}(0), \frac{S h_{x}}{\sqrt{R e_{x}}}=-\phi^{\prime}(0)$,

\section{CONFIRMATION OF NUMERICAL UPSHOTS}

The moderate Runge-Kutta-Fehlberg (RKF-45) with technique of shooting is applied to explain the highly nonlinear joined equations [12, 13, 14] along with the associated boundary conditions [15\&16]. Table.1 shows the assessment of present outcomes with that of previous outcomes, where we can observe exact agreement.

Table 1: Assesment of $-\theta^{\prime}(0)$ for values of Pr when $M=\beta=R=$

$$
N=\beta_{1}=S c=\gamma=0 .
$$

\begin{tabular}{|c|c|c|c|c|}
\hline $\operatorname{Pr}$ & $\begin{array}{c}\text { Magyari and } \\
\text { Keller [42] }\end{array}$ & $\begin{array}{c}\text { Bidin and Nazar } \\
{[43]}\end{array}$ & $\begin{array}{c}\text { Ishak } \\
{[44]}\end{array}$ & $\begin{array}{c}\text { Present } \\
\text { results }\end{array}$ \\
\hline 1 & 0.9548 & 0.9547 & 0.9548 & 0.9549 \\
\hline 2 & & 1.4714 & 1.4715 & 1.4714 \\
\hline 3 & 1.8691 & 1.8691 & 1.8691 & 1.8691 \\
\hline 5 & 2.5001 & & 2.5001 & 2.5001 \\
\hline 10 & 3.6604 & & 3.6604 & 3.6604 \\
\hline
\end{tabular}

\section{GRAPHICAL ILLUSTRATION}

\subsection{Performance of $M, \beta, R, N$ on $f^{\prime}(\eta) \&$ Performance of $R$ on $\boldsymbol{f}^{\prime \prime}(\boldsymbol{\eta})$}

From Fig. 2 it is witnessed that as $M$ reaches higher values there is a quick reduction in velocity due to Lorentz force which compete against the motion of the fluid and tends to reduce fluid flow. Advanced values of $\beta$ enhances fluid viscosity and slows down the motion of the fluid as shown in Fig. 3. Impact of $\mathrm{R}$ on $f^{\prime}(\eta)$ and $f^{\prime \prime}(\eta)$ is shown in Fig.4 \& Fig.5. Higher values of R ie, higher buoyancy forces have an enhancing effects on velocity since it acts as a pressure gradient which accelerates the fluid with in the boundary layer. Furthermore shear stress increases in the vicinity of the boundary $0 \leq \eta \leq 0.5556$ and decreases within the fluid domain $\eta>0.5556$ for an increase in R. Solutal buoyancy number $\mathrm{N}$ acts as favorable case for velocity and this can be observed from Fig. 6.

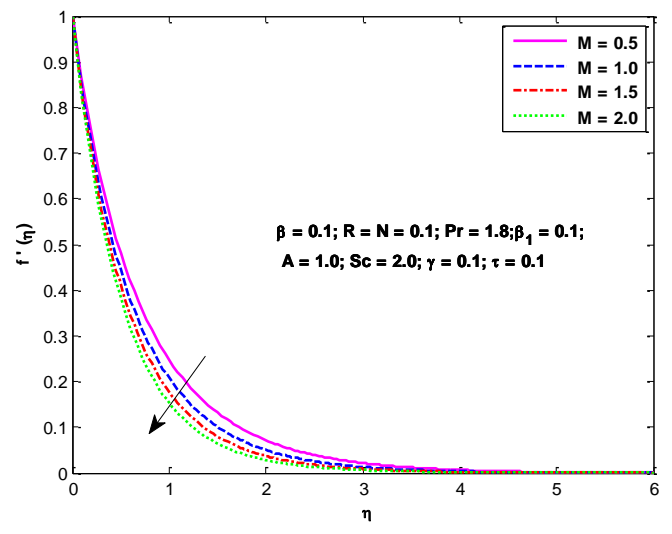

Fig. 2 Dominance of $M$ on $f^{\prime}(\eta$ 


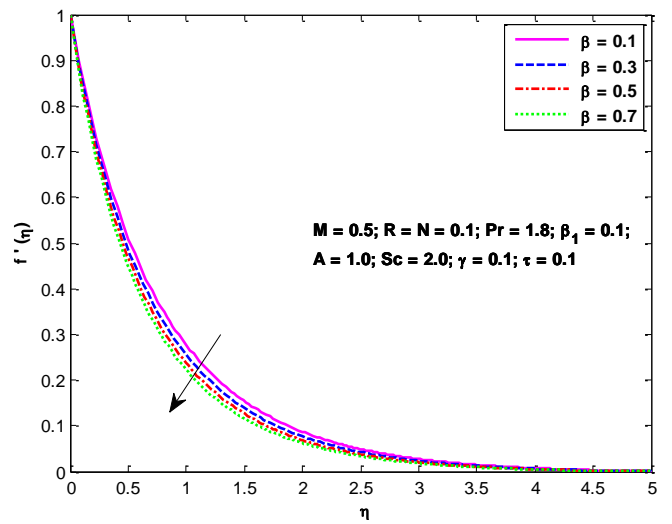

Fig. 3 Dominance of $\beta$ on $f^{\prime}(\eta)$

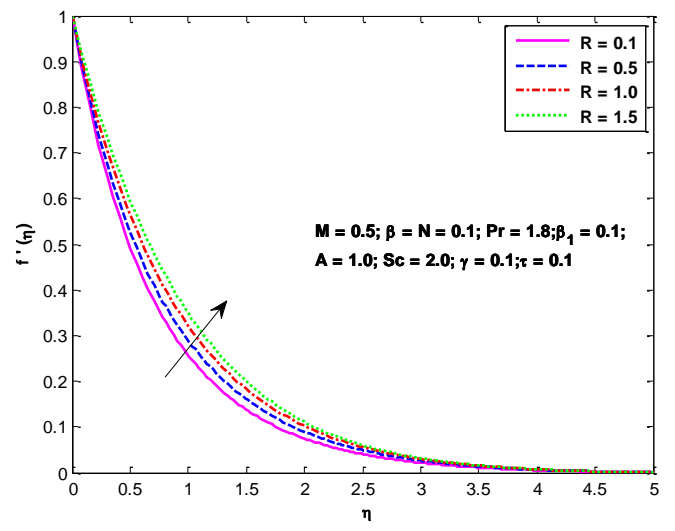

Fig. 4 Dominance of $\mathrm{R}$ on $f^{\prime}(\eta)$

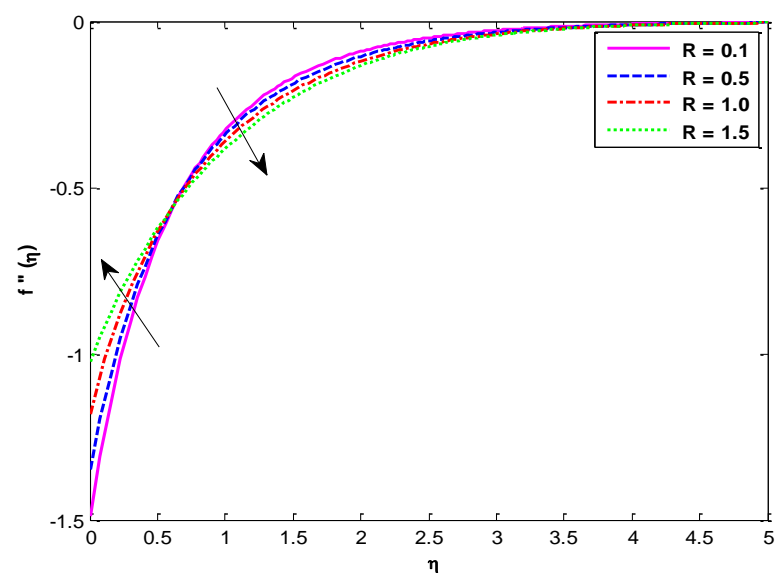

Fig. 5 Dominance of $\mathrm{R}$ on $f^{\prime \prime}(\eta)$

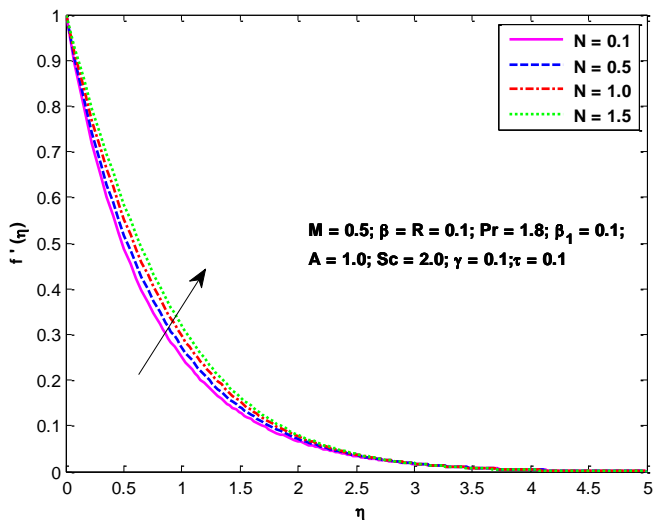

Fig. 6 Dominance of $\mathrm{N}$ on $f^{\prime}(\eta)$

\subsection{Performance of $\beta_{1}, \operatorname{Pr}, A$ on $\theta(\eta)$}

Advanced values of thermal relaxation time $\beta_{1}$ weaken the thickness of the boundary layer across the fluid and as a result temperature diminishes. This physical phenomenon best explains Cattaneo- Christov heat flux relaxation in Fig.7. Minor values of Pr yield thicker boundary layer with higher temperature across the boundary layer. Thermal boundary layer is generated within the lower part of the boundary layer at higher $\mathrm{Pr}$, as a result temperature gradient vanishes adjacent to the free surface as illustrated in Fig. 8. It is interesting to observe an over shoot of temperature in the fluid adjacent to the boundary that occurs for some negative values of $A$ and hence heat is expected to flow towards the wall from the ambient fluid and later the temperature exponentially decreases and approaches the free stream condition. This means that for some negative ' $A$ ' one expects reverse heat flow in the viscinity of the sheet. This result can be observed in many consistent previous results. This is known as Sparrow-Gregg-Hill (SGH) phenomenon which is observed in Fig. 9.

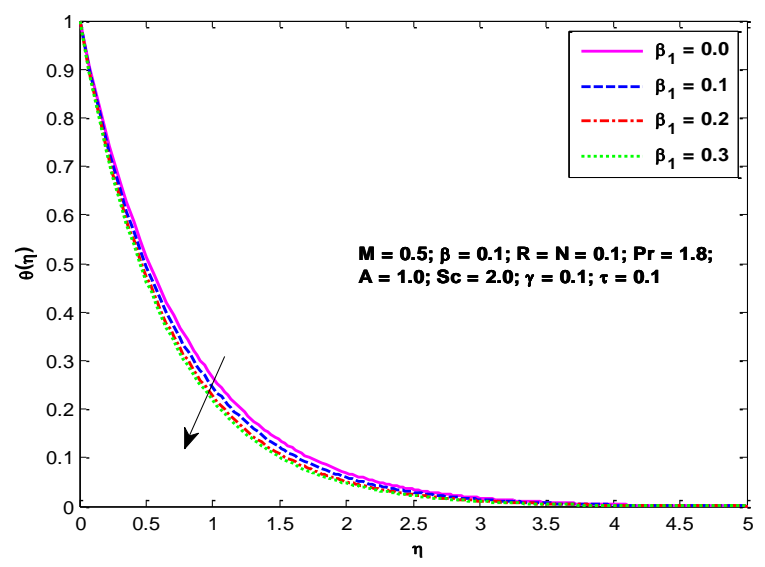

Fig. 7 Dominance of $\beta_{1}$ on $\theta(\eta$ 


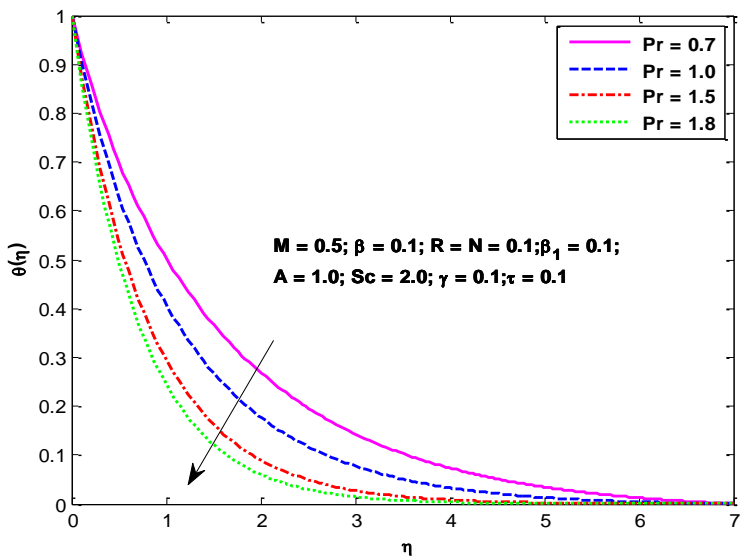

Fig. 8 Dominance of $\operatorname{Pr}$ on $\theta(\eta)$

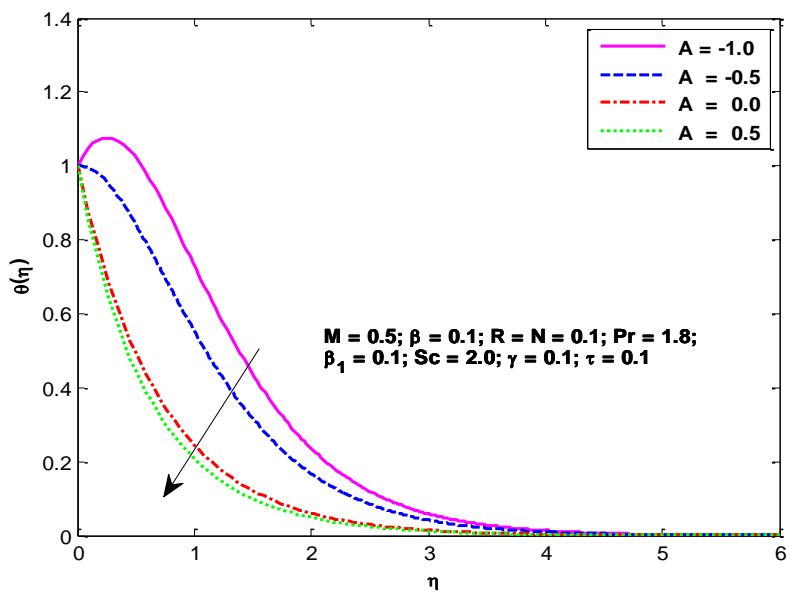

Fig. 9 Dominance of A on $\theta(\eta)$

\section{3. Performance of $S c, \gamma, \tau$ on $\varphi(\eta)$}

The concentration gets reduced for increasing value of Sc. This may be explained as larger values of Sc corresponds to smaller values of mass diffusivity and hence thickness of solutal boundary layer becomes thinner when Sc is large as illustrated in Fig.10. Chemical reaction parameter $\gamma$ increases interfacial mass transfer and reduces the solutal boundary layer. This results in weaker molecular diffusivity and thinner boundary layer. Therefore advanced values of $\gamma$ reduces $\boldsymbol{\varphi}(\boldsymbol{\eta})$ which is illustrated in Fig. 11. Influence of thermophoretic parameter $\tau$ on concentration is plotted in Fig. 12. It is observed that the effect of increasing value of $\tau$ gradually decreases species concentration.

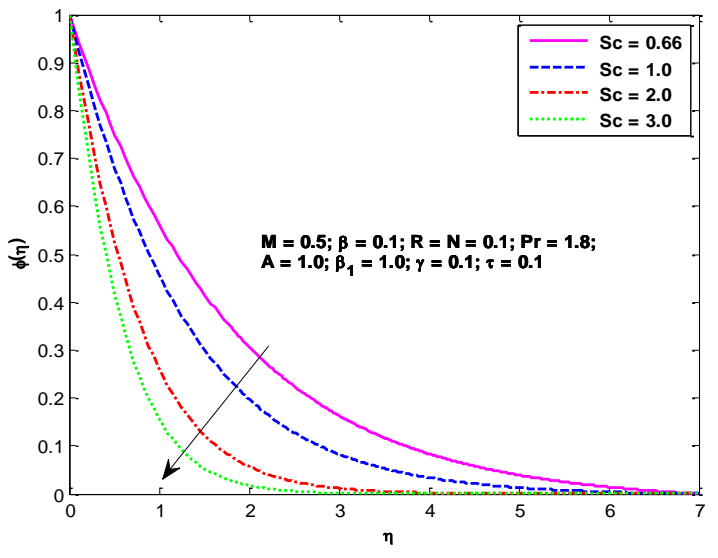

Fig. 10 Dominance of $S c$ on $\phi(\eta)$

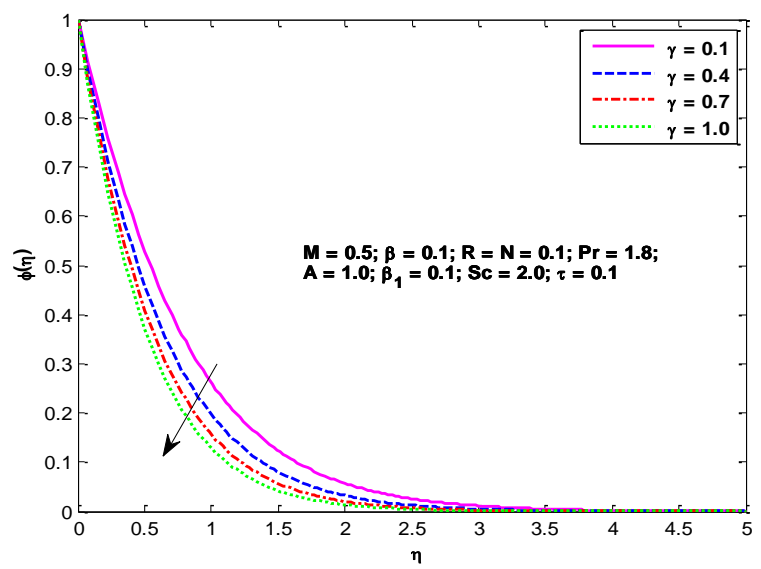

Fig. 11 Dominance of $\gamma$ on $\phi(\eta)$

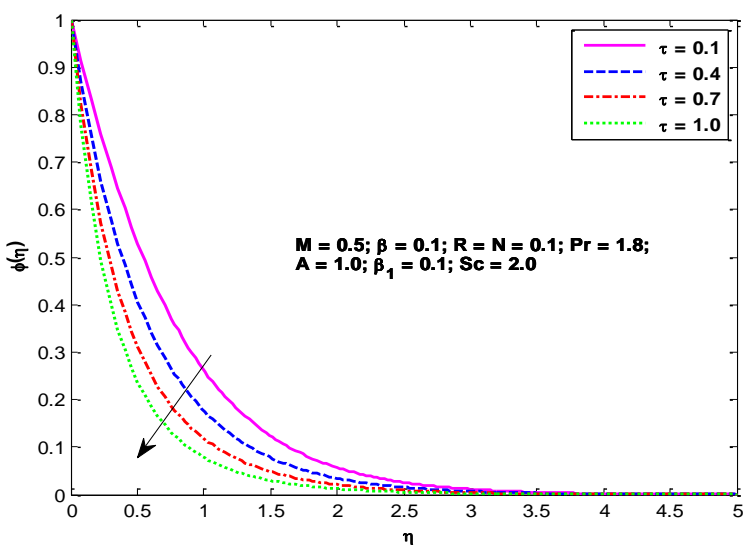

Fig. 12 Dominance of $\tau$ on $\phi(\eta)$ 


\subsection{Performance of skin friction coefficient, Nusselt number, Sherwood number}

The relation among surface drag coefficient, $\mathbf{M}$, and $\beta$ in the range from 0.1 to 1 is plotted in Fig. 13. As M reaches from 0.1 to 1 surface drag coefficient gets the form of straight lines with negative slope which means that for higher $\mathrm{M}$ it gradually decreases. It is also seen that for higher Maxwell parameter $\beta$ both the parameters declines. The mutual relation between $\mathrm{R}, \mathrm{N}$ and skin friction coefficient is graphically presented in Fig.14. It is noticed that there is a negligible increase in the drag coefficient when $R=0.1$ as $\mathrm{N}$ increases from 0.1 to 1 . As $\mathrm{R}$ takes higher values it is seen that there is a monotonic increase in the drag coefficient for the same range of variation of N. In Fig. 15 Nusselt number versus Prandtl number $\operatorname{Pr}$ for a variation of thermal relaxation parameter $\beta_{1}$ is drawn. The profiles of Nusselt number are straight lines with positive slope indicating that the rate of heat transfer increases with increasing Prandtl number. As $\beta_{1}$ takes higher values the gradient of the profile increases and also with increase in Pr indicating an enhancement in the rate of heat transfer. Figure. 16 is the graph of Sherwood number drawn for a variation in chemical reaction parameter $\gamma$ and Schmidt's number Sc. It is observed that as $\gamma$ increases there is a steady growth in Sherwood number. Increment in Sc leads to a significant enhancement in Sherwood number and the corresponding profiles are parallel.

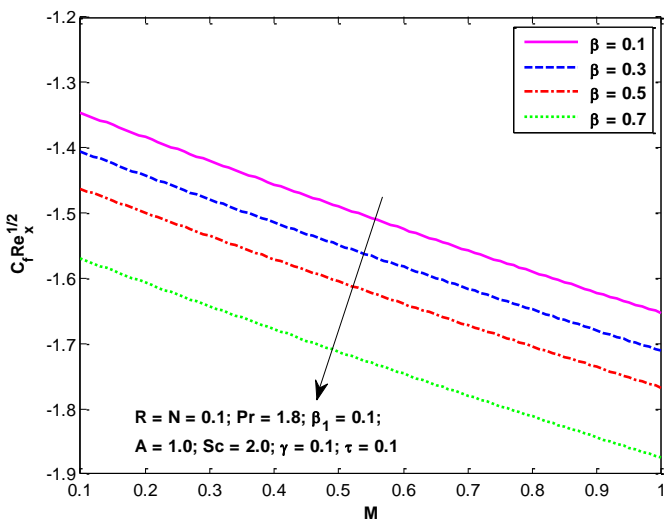

Fig. 13 Variation of Skin friction with $\beta$ for $\mathrm{M}$

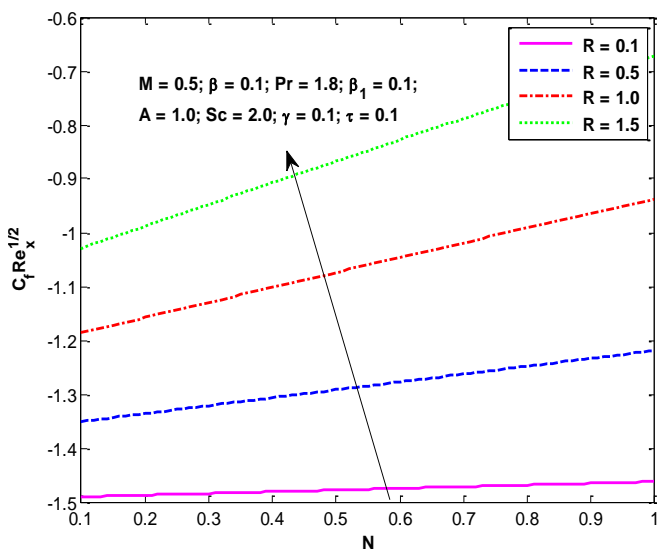

Fig. 14 Variation of Skin friction with $\mathrm{R}$ for $\mathrm{N}$

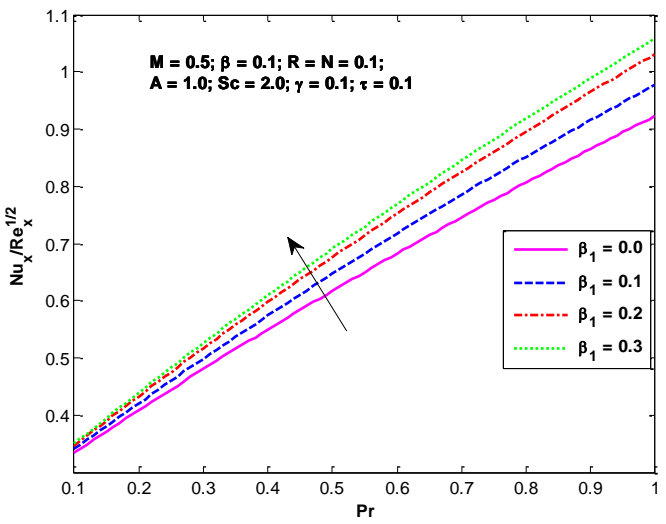

Fig. 15 Variation of Nusselt number with $\beta_{1}$ for $\operatorname{Pr}$

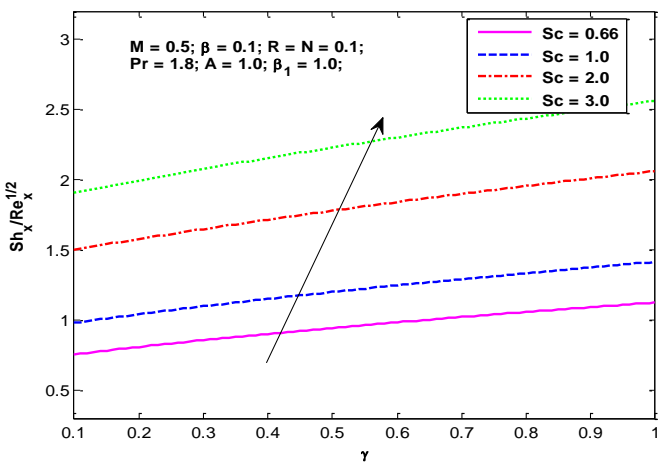

Fig. 16 Variation of Sherwood number with Sc for $\gamma$

\section{CONCLUSIONS}

The steady boundary layer flow of an incompressible, electrically conducting Maxwell fluid over an exponentially stretching sheet in the presence of Cattaneo-Christov heat flux and chemically reactive solute transfer with first order chemical reaction is analyzed. The following conclusions are drawn:

$>$ Flow deceleration is observed with increasing Maxwell parameter $\beta$ and magnetic field intensity $M$.

$>\quad$ The influence of themophorotic parameter $\tau$ is clearly observed on concentration, as it increases concentration decreases.

$>$ Both thermal and solutal buoyancy forces accelerates velocity gradients.

$>$ Sparrow-Gregg-Hill (SGH) phenomenon is observed with the temperature exponent.

$>\quad$ The temperature and concentration are enhanced with increasing magnetic field $\mathrm{M}$ and Maxwell parameter $\beta$.

$>$ Temperature in the Cattaneo-Christov model is seen to be lesser than that of the Fourier model.

$>$ Suppression in velocity due to Maxwell and magnetic field parameters and a reduction in surface drag coefficient are observed.

$>\quad$ The mass transfer rate increases with Schmidt number and chemical reaction parameter 


\section{NOMENCLATURE}

$\begin{array}{cl}\mathrm{g} & \text { Acceleration due to gravity } \\ k_{0} & \text { Chemical reaction } \\ \beta_{T} & \text { Coefficient of thermal expansion } \\ \beta_{C} & \text { Coefficient of concentration expansion } \\ \rho & \text { Density of the fluid } \\ \sigma & \text { Electrical conductivity } \\ C_{\infty} & \text { Free stream concentration assumed to be constant } \\ T_{\infty} & \text { Free stream temperature assumed to be constant } \\ T_{0} & \text { Heating/cooling reference temperature } \\ v & \text { Kinematic viscosity } \\ C^{*} & \text { Local fluid concentration } \\ T^{*} & \text { Local fluid temperature } \\ \mathrm{D} & \text { mass diffusivity } \\ \lambda & \text { Relaxation time of the fluid } \\ \lambda_{1} & \text { Relaxation time for heat flux } \\ C_{0} & \text { Reference concentration } \\ T_{r} & \text { Reference temperature } \\ c_{p} & \text { Specific heat at constant pressure } \\ k & \text { Thermal conductivity } \\ k_{r} & \text { Thermophoretic coefficient. } \\ \bar{u} & \text { Velocity component along x-axis } \\ \bar{v} & \text { Velocity component along y-axis } \\ & \end{array}$

\section{REFERENCES}

Ashwin kumar G.P., Sulochana C., 2018, "Effect of radiation absorption and buoyancy force on the MHD mixed convection flow of Casson nanofluid embedded with Al50Cu50 alloy nanoparticles", Multidiscipline Modeling in Materials and Structures, 14(5),1082-1100.

https://doi.org/10.1108/MMMS-12-2017-0164

Awais, M., Awan, S., Iqbal, K., Ashfaq Khan, Z., Zahoor Raja, M,A., 2018“Hydromagnetic mixed convective flow over a wall with variable thickness and Cattaneo-Christov heat flux model OHAM analysis", Results in Physics. 8,621-627.

https://doi.org/10.1016/j.rinp.2017.12.043

Cattaneo, C Sulla conduzione del calore, "Atti Semin Mat Fis Univ Modena Reggio” Emilia 3(1948), 83-101

Christov, C, I.,(2009), "On frame indifferent formulation of the MaxwellCattaneo model of finite-speed heat conduction", Mechanics Research Communications. 36, 481-486.

https://doi.org/10.1016/j.mechrescom.2008.11.003

Fetecau, C., 2003, "A new exact solution for the flow of Maxwell fluid past an infinite plate", Int. J. Nonlin. Mech., 38(3), 423-427. https://doi.org/10.1016/S0020-7462(01)00062-2

Fick A, Uber diffusion. Ann Phys. (1855), 170:59-86. https://doi.org/10.1002/andp.18551700105
Fourier J, B, J., (1822), “Théorie Analytique De La Chaleur, Paris”.

Gireesha, B.J., Mahanthesh, B., Subba, R., Gorla, R., Krupalakshmi, K.L, 2018, "Mixed convection two-phase flow of Maxwell fluid under the influence of non-linear thermal radiation, non-uniform heat source/sink and fluid-particle suspension", A in Shams engineering journal., 9, 735746.

https://doi.org/10.1016/j.asej.2016.04.020

Hady, F, M., Ibrahim, F, S., Abdel-Gaied, S, M., \& Eid, M. R., 2011," Effect of heat generation/absorption on natural convective boundary-layer flow from a vertical cone embedded in a porous medium filled with a non-Newtonian nanofluid", International Communications in Heat and Mass Transfer, 38(10), 1414-1420

https://doi.org/10.1016/j.icheatmasstransfer.2011.07.008

Hayat,T., T. Muhammad, T., A. Alsaedi, A., Ahmad, B., 2016, "Threedimensional flow of nanofluid with Cattaneo-Christov double diffusion", Results in Physics. 6, 897-903. https://doi.org/10.1016/j.rinp.2016.10.017

Lin Y., Zheng L., Zhang X., Ma L., Chen G., 2015, "MHD pseudo-plastic nano fluid unsteady flow and heat transfer in a finite thin film over stretching surface with internal heat generation," Int J Heat Mass Transf. 84,903-911.

https://doi.org/10.1016/j.ijheatmasstransfer.2015.01.099

Loganathan, P., Arasu, P. P., 2010, "Thermophoresis effects on nonDarcy MHD mixed convective heat and mass transfer past a porous wedge in the presence of suction/injection.Theoretic Applied Mechanics, 37(3), 203-227

https://doi.org/10.2298/TAM1003203L

Nagalakshmi, P. S, S., Vijaya, N., 2020, "MHD flow of Carreau Nanofluid explored using CNT over a nonlinear stretched sheet", Frontiers in Heat and Mass Transfer (FHMT), 14, 4. http://dx.doi.org/doi: 10.5098/hmt.14.4

Rahbari, A., Abbasi, M., Rahimipetroudi, I., Sundén, B., Ganji, D, D., M. Gholami, M., 2018, "Heat transfer and MHD flow of non-Newtonian Maxwell fluid through a parallel plate channel", Mech Sci. 9, 61-70 https://doi.org/10.5194/ms-9-61-2018

Ramesh, G,K., Gireesha, B,J., Bagewadi., C,S., 2012, "MHD flow of a dusty fluid near the stagnation point over a permeable stretching sheet with non-uniform source/sink”, Int J Heat Mass Transf. 55: 4900-4907. https://doi.org/10.1016/j.ijheatmasstransfer.2012.05.003

Rawat, S. K., Pandey, A. K., \& Kumar, M., 2018, "Effects of chemical reaction and slip in the boundary layer of MHD nanofluid flow through a semi-infinite stretching sheet with thermophoresis and Brownian motion, The Lie group analysis. Nanoscience and Technology: An International Journal, 9(1), 47-6.

https://doi.org/10.1615/NanoSciTechnolIntJ.2018025363

Rout, B,R., S.K.Parida, S,K., Panda, S., 2013, "MHD Heat and Mass Transfer of Chemical Reaction Fluid Flow over a Moving Vertical Plate in Presence of Heat Source with Convective Surface Boundary Condition", International Journal of ChemicalEngineering.ArticleID296834, https://doi.org/10.1155/2013/296834 
Shateyi, S., 2008, "Thermal Radiation and Buoyancy Effects on Heat and Mass Transfer over a Semi-Infinite Stretching Surface with Suction and Blowing”, Journal of Applied Mathematics, Article ID 414830, 12 page. https://doi.org/10.1155/2008/414830

Sui, J., L. Zheng, 1., Xinxin, Z., 2016, "Boundary layer heat and mass transfer with Cattaneo-Christov double-diffusion in upper convected Maxwell nanofluid past a stretching sheet with slip velocity", International Journal of Thermal Sciences, 104, 461-468.

https://doi.org/10.1016/j.ijthermalsci.2016.02.007

Umer Farooq., Dianchen Lu., Shahzad Munir., Muhammad Ramzan,., Muhammad Suleman \& Shahid Hussain., 2019, "MHD flow of Maxwell fluid with nano materials due to an exponentially stretching surface", Scientific reports. 9,9312.

https://doi.org/10.1038/s41598-019-43549-0

Vajravelu, K., Li, R., Dewa surendra, M., Benarroch, J., Ossi, N., Zhang, Y., M. Sammarco,M., Prasad, K,V., 2017, "Analysis of MHD boundary layer flow of an Upper- Convected Maxwell fluid with homogeneousheterogeneous chemical reactions", Commun. Numerical Analysis.2, 202216 .

https://doi.org/10.5899/2017/cna-00324

Vedavathi N., Dharmaiah G., Balamurugan K.S., Prakash J. (2017), 'Heat transfer on mhd nanofluid flow over a semi infinite flat plate embedded in a porous medium with radiation absorption, heat source and diffusion thermo effect', Frontiers in Heat and Mass Transfer, 9(38) (2017), 1-8 https://doi.org/10.5098/hmt.9.38
Vijaya, N., Venkata Ramana Reddy, G., Hari Krishna, Y., 2019, "NonAligned Stagnation Point Flow of a Casson Fluid Past a Stretching Sheet in a Doubly Stratified Medium", Fluid Dynamics \& Materials Processing, 15(3), 233-251.

https://doi.org /10.32604/fdmp.2019.037

Vijaya, N., Sunil Babu, G., Vellanki Lakshmi, N., 2020, "influence of critical parameters on liquid thin film flow of casson nano fluid over elongated sheet under thermophorosis and brownian motion", Frontiers in Heat and Mass Transfer (FHMT), 15(23),

https://doi.org/10.5098/hmt.15.23

Vijaya, N., Arifuzzaman, S,M., Raghavendra Sai , Ch. Manikya Rao.,(2020), "analysis of arrhenius activation energy in electrically conducting casson fluid flow induced dueto permeable elongated sheet with chemical reaction and viscous dissipation", Frontiers in Heat and Mass Transfer (FHMT), 15(26).

https://doi.org/10.5098/hmt.15.26

Webshet Ibrahim,, Mekonnen Negera., 2020, "MHD slip flow of upperconvected Maxwell nanofluid over a stretching sheet with chemical reaction ', Journal of the Egyptian Mathematical Society, 28:7. https://doi.org/10.1186/s42787-019-0057-2

Yahaya Shgiya Daniel., Simon K. Daniel., 2015, "Effects of buoyancy and thermal radiation on MHD flow over a stretching porous sheet using homotopy analysis method", Alexandria Engineering Journal, 54(2015),705-712.

https://doi.org/10.1016/j.aej.2015.03.029 